Article

\title{
Construction of Knowledge Graphs for Maritime Dangerous Goods
}

\author{
Qi Zhang ${ }^{1}{ }^{1}$, Yuanqiao Wen ${ }^{2,3,4, *}$, Chunhui Zhou ${ }^{1,3,4, *}$, Hai Long ${ }^{5}$, Dong Han ${ }^{1}$, Fan Zhang $1,3,4$ \\ and Changshi Xiao ${ }^{1,3,4}$ \\ 1 School of Navigation, Wuhan University of Technology, Wuhan 430063, China; zhangqiwhut@163.com (Q.Z.); \\ handong@whut.edu.cn (D.H.); michael_zf@whut.edu.cn (F.Z.); cs_xiao@hotmail.com (C.X.) \\ 2 Intelligent Transportation System Research Centre, Wuhan University of Technology, Wuhan 430063, China \\ 3 National Engineering Research Centre for Water Transport Safety, Wuhan 430063, China \\ 4 Hubei Key Laboratory of Inland Shipping Technology, Wuhan 430063, China \\ 5 Institute of Medical Informatics, Statistics and Epidemiology (IMISE), University of Leipzig, \\ 04107 Leipzig, Germany; longhail2008@yahoo.de \\ * Correspondence: wenyqwhut@foxmail.com (Y.W.); chunhui@whut.edu.cn (C.Z.)
}

Received: 16 April 2019; Accepted: 16 May 2019; Published: 19 May 2019

\begin{abstract}
Dangerous goods occupy an important proportion in international shipping, and government and enterprises pay a lot of attention to transport safety. There are a wide variety of dangerous goods, and the knowledge involved is extensive and complex. Organizing and managing this knowledge plays an important role in the safe transportation of dangerous goods. The knowledge graph is a mass of brand-new knowledge management technologies that provide powerful technical support for integrating domain knowledge and solving the problem of the "knowledge island." This paper first introduces the knowledge of maritime dangerous goods (MDG); constructs a three-layer knowledge structure of MDG, dividing this knowledge into two categories; uses ontology to express the concepts, entities, and relations of MDG; and puts forward the representation methods of the conceptual layer and entity layer and designs them in detail. Finally, the knowledge graph of maritime dangerous goods (KGMDG) is constructed. Furthermore, we demonstrate the knowledge visualization, retrieval, and automatic judgment of segregation requirement based on KGMDG. It is proved that KGMDG does not only help to simplify the retrieval process of professional knowledge and to promote intelligent transportation but is also conducive to the sharing, dissemination, and utilization of MDG knowledge.
\end{abstract}

Keywords: knowledge graph; maritime dangerous goods; ontology; knowledge representation; knowledge management

\section{Introduction}

Waterway transportation, with the characteristics of large capacity and low cost, is the main transportation method for social production and industrial development. According to statistics, $10 \%-15 \%$ of the goods transported by water are dangerous goods [1]. Due to the continuous progress of modern production technology and tools, the types and quantities of chemicals needed are increasing. Therefore, the categories and quantities of dangerous goods transported by water are also increasing [2]. With the rapid increase in dangerous goods transportation, the accidents during transportation become more and more frequent. According to statistics from 1998-2008, about 15\% of the world's container ship accidents were caused by dangerous goods [3], including a large number of casualties, property losses, and irreversible environmental pollution. For example, on 14 July 2012, a fire and explosion broke out on the container ship "MSC Flaminia" on its way from Charleston to Antwerp, Belgium, killing a total of three people. The accident investigation report showed that the accident was caused 
by improper loading of divinylbenzene. On 13 February 2017, a fire broke out on "APL AUSTRIA", a 6350 TEU container ship owned by American President Lines. The accident was caused by a chemical reaction of calcium hypochlorite.

The transportation chain of maritime dangerous goods (MDG) is a complex system involving many factors, such as human, ship, cargo, environment, and management, and the knowledge involved is very complicated. At present, the transportation of MDG is mainly carried out in strict accordance to the International Maritime Dangerous Goods Code (IMDG Code). The IMDG Code divides dangerous goods into nine categories and specifies in detail the packaging, stowage, segregation, consignment procedures, emergency operations, and so on [4]. In actual transportation, due to the strict loading conditions, scattered knowledge of MDG, cumbersome inquiry procedures, and complicated judgment of stowage and segregation, violations often occur.

With the development of the information age, the management technology of huge system knowledge is constantly updated. The ontology [5] and semantic web [6] can correlate a large amount of knowledge and information through certain organizational forms, and it also has a certain ability of knowledge reasoning. Therefore, knowledge management technologies, such as ontology and semantic web are being applied to more and more industries [7-10]. Especially in recent years, the knowledge graph [11] developed from the semantic web is attracting more and more researchers' attention.

The knowledge graph is a mass new-mode knowledge management and service based on big data [12]. It is a semantic network knowledge base with a directed graph structure [13]. By extracting information from semi-structured or unstructured data to form triples such as (subject, predicate, object), a knowledge graph can realize rapid knowledge response and reasoning [14]. It can display the complicated relations between professional knowledge, connect fragmentary knowledge, and it can support knowledge retrieval, knowledge question and answer, knowledge recommendation, knowledge visualization, and other applications. This provides a technology to solve the problem of the "knowledge island" in the field of MDG. This paper introduces the concept system of MDG, searches and integrates the industry knowledge, and constructs the knowledge graph of maritime dangerous goods (KGMDG). The KGMDG realizes the relationship in dangerous goods knowledge and has important reference and service value for users such as government and enterprises.

\section{The Objectives}

The objectives of this study were (1) to organize and classify the knowledge of MDG; (2) to depict the core concepts, entities, and their relationship of MDG; and (3) to construct the KGMDG based on the above research.

\section{Literature Review}

The research on the safe transportation of dangerous goods mainly focuses on risk management and control. There are many risks in the transportation process of dangerous goods, so it is very important to establish an effective risk quantification and management mechanism for shipping companies. Lam et al. used a rough set approach to classify and judge the safety attributes of vessels and cargo, and established a risk assessment system for MDG, so as to formulate a risk reduction strategy [15]. According to the dynamic changes of dangerous sources in the chemical storage area, Liu et al. proposed a dynamic hazard analysis method and a classification system and developed a decision support system for the emergency response regarding dangerous goods based on the database [16]. Risk management of dangerous goods transportation is a system consisting of risk identification, evaluation, and control. Based on this, Yang et al. proposed a framework for a quality function under a fuzzy environment, which can effectively control the risk in the process of dangerous goods transportation [17]. Juniora et al. focused on the risk management in cargo transportation and proposed a method to select different cargo transportation companies according to their technical preferences, in order to improve the safety of dangerous cargo transportation [18]. Vlies et al. put forward a qualitative risk assessment method for dangerous goods through seven case studies in 
the Netherlands. This method can effectively control the external safety risks related to waterway transportation [19]. The research above constructs decision-making to reduce risks by analyzing the risks in the process of dangerous goods transportation. However, the transportation of dangerous goods involves a lot of knowledge. How to associate that knowledge and realize the correlation between the goods is of great significance for reducing risks and ensuring transport safety.

At present, the research productions of knowledge graph are mostly general-purpose knowledge graph. In addition to Google, the famous general-purpose knowledge graphs include DBpedia [20], YAGO [21], Freebase [22], and so on. These knowledge graphs include hundreds of millions of entities and relations. Google knowledge graph, for example, includes 500 million entities and 2.5 billion relations. Although the research on knowledge graphs in China started late, there are also some products, such as Baidu's knowledge graph, Sogou's knowledge graph, "Zhishi.me" [23], and "CN-DBpedia" constructed by Fudan University [24], etc. The general-purpose knowledge graph has characteristics of large scale, wide breadth, and a high degree of automation and openness. However, its research depth is shallow, the accuracy is relatively low, and the degree of reasoning is weak, so it is difficult to directly use in a specific industry.

Compared with the general-purpose knowledge graph, domain-specific knowledge graphs involve a specific industry. They are oriented to specific industry objects, have a deeper research depth, and higher accuracy. At present, there is no research on the construction of knowledge graph in the field of dangerous goods. However, there are many research findings in other industries, such as the field of medicine. For example, the well-known domain-specific knowledge graphs in foreign countries include the "Knowlife" [25] and the knowledge graph of disease symptoms [26]. In recent years, China's domain-specific knowledge graph has achieved some research productions. For example, the knowledge graph of traditional Chinese medicine published by the China Academy of Chinese Medical Sciences, which contains more than 600,000 entities and more than 12.7 million relations [27], includes the knowledge graph of traditional Chinese medicine prescription [28]; the "HKDP," which is the knowledge graph of pediatric disease prevention system [29]; etc. Other industries also have some achievements, such as the academic knowledge graph "AceKG" [30], published by Shanghai Jiao Tong University; the educational knowledge graph "KnowEdu" [31]; and so on. Because of the need for specific industry data and the high dependence on industry experts, the construction of this knowledge graph is relatively difficult and slow.

\section{Materials and Methods}

This section introduces the methods of construction of the KGMDG. We first combed the knowledge of MDG and classified them; then studied the core concepts, entities, and their relations of MDG; and finally designed the conceptual layer and the entity layer to complete the construction of the graph.

\subsection{Knowledge of Maritime Dangerous Goods}

\subsubsection{Knowledge Analysis of Maritime Dangerous Goods Based on Multi-Granularity}

The knowledge involved in MDG cannot be fully understood and summarized by a single granularity description, and it needs to be measured from the point of view of "coarse" and "fine." Fine-grained knowledge is a single, indivisible knowledge. Coarse-grained knowledge, from the macro point of view, is general and rough knowledge. As shown in Figure 1, the knowledge structure of MDG is an organic whole composed of knowledge of different granularities. We regard this knowledge structure as a pyramid structure composed of knowledge source, core layer, middle layer, and basic layer, and the knowledge granularity changes from coarse to fine layer by layer. The knowledge among different layers is interrelated, and the knowledge structure of the lower layer supports the upper layer.

The knowledge layer and its relations are as follows:

Knowledge source: The knowledge involved in the MDG mainly comes from international covenants, domestic regulations, and some technical standards. Among them, the IMDG Code, which 
specifies the packaging, labeling, stowage, segregation, handling, and emergency operation, is the most important international covenant in the transportation chain of MDG. Any international ship carrying dangerous goods must enforce it. Meanwhile, it plays an important role in protecting crews and reducing marine pollution. In addition, other knowledge includes the Material Safety Data Sheet (MSDS), People's Republic of China Security Supervision and Administration For Shipping Dangerous Goods Regulations, and so on.

Core layer (substructure): This layer is composed of different operation requirements in different stages of transportation, storage, and loading of MDG. It is the core content of the knowledge structure, which can reflect the key research contents and development trends of this field. The knowledge granularity of this layer can be expressed as:

$$
G_{c l}=\left\{G_{c l 1}, G_{c l 2}, \cdots, G_{c l m}\right\}, m>1
$$

where $G_{c l}$ is the core layer knowledge and $G_{c l m}$ is a certain knowledge stage.

Middle layer (essential element): Based on the core layer, this layer consists of packaging, stowage, segregation, and other operational requirements. They are the main research content and important knowledge components, and it can reflect the main characteristics of MDG knowledge. The knowledge granularity of this layer can be expressed as:

$$
G_{m l}=\left\{G_{m l 1}, G_{m l 2}, \cdots, G_{m l m}\right\}, m>1
$$

where $G_{m l}$ is the middle layer knowledge and $G_{m l m}$ is a specific operational requirement.

Basic layer (knowledge element): Based on the middle layer, it consists of the attributes of dangerous goods, including the classification, United Nations number (UN number), subsidiary risks, physical and chemical properties, toxicity, and so on. The knowledge is the most basic and the smallest units in the field of MDG, and it also reflects the nature and characteristics of MDG. The knowledge granularity of this layer can be expressed as:

$$
G_{b l}=\left\{G_{b l 1}, G_{b l 2}, \cdots, G_{b l m}\right\}, m>1
$$

where $G_{b l}$ is the basic layer knowledge and $G_{b l m}$ is an attribute of dangerous goods.

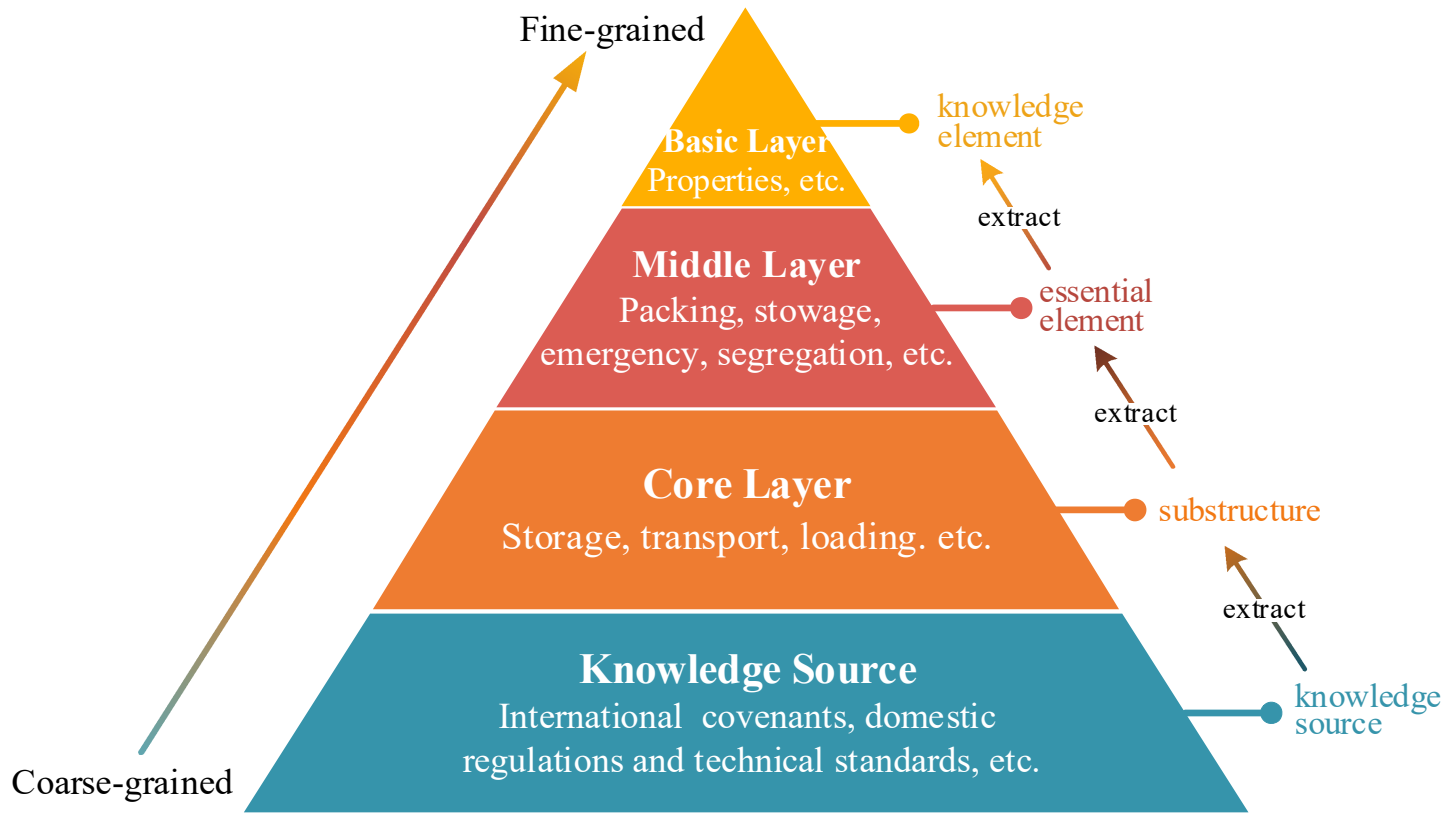

Figure 1. Knowledge structure of MDG. 
4.1.2. Knowledge Classification of Maritime Dangerous Goods Based on Knowledge Representation

To facilitate knowledge representation, we needed to classify structured and unstructured knowledge before constructing the knowledge graph. We divided knowledge of MDG into direct representation knowledge and indirect representation knowledge according to the form of knowledge representation, as shown in Figure 2.

Direct representation knowledge: This part of knowledge can be expressed directly by ontology, including the essential attributes (physical and chemical properties, toxicity), special attributes (category, UN number, subsidiary risks), and other knowledge (packing, packing group, limited quantity, excepted quantity).

Indirect representation knowledge: This part of knowledge is not only an important research content in this field but also a difficult point in knowledge representation. Because this part of knowledge cannot be expressed directly by ontology, we needed to define the corresponding rules to get it. This part includes cargo stowage, segregation, emergency, and other operations.

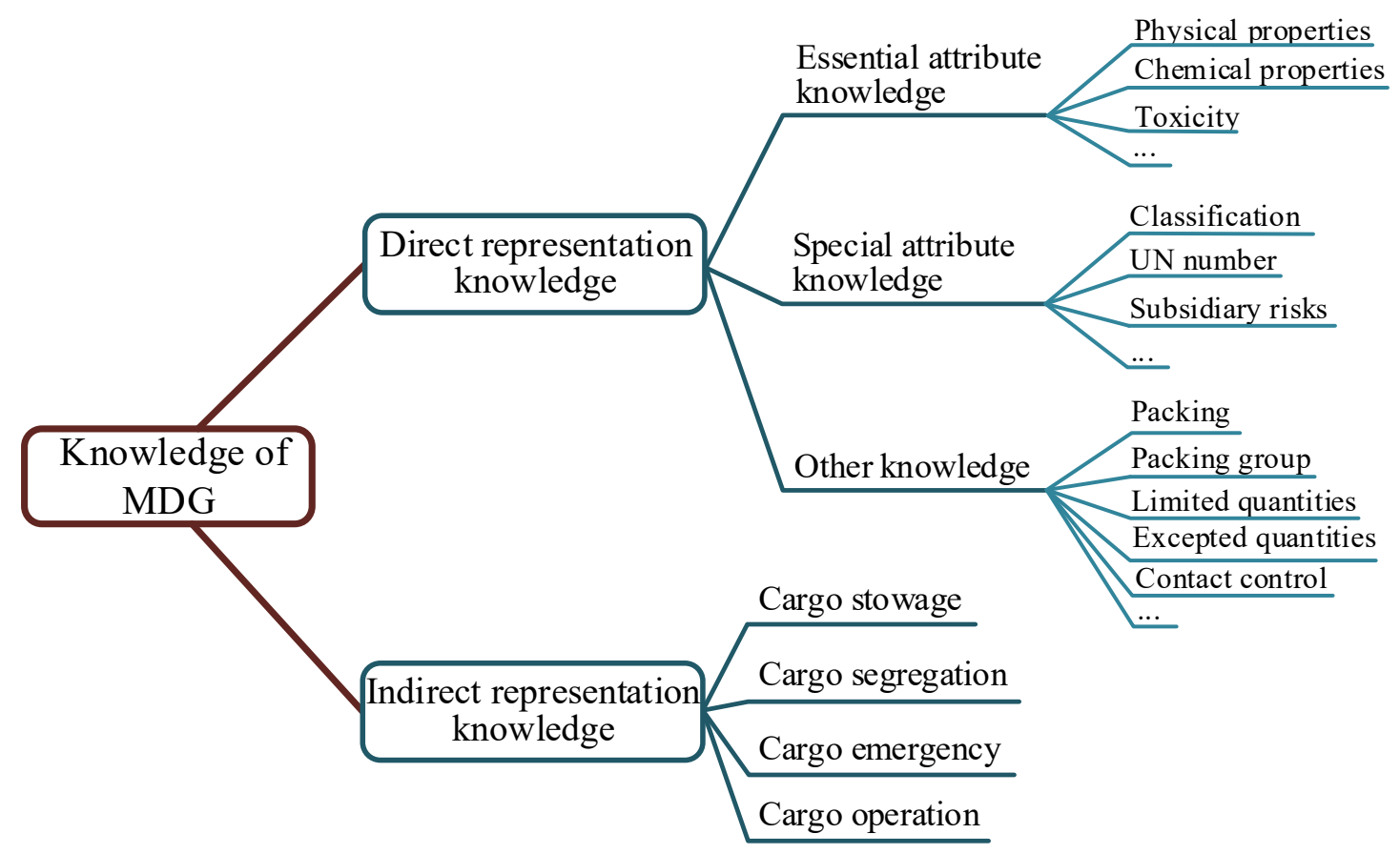

Figure 2. Knowledge classification of MDG.

\subsubsection{Knowledge Representation of Maritime Dangerous Goods}

Knowledge representation is to encode objective knowledge of the human world so that computers can understand and process it [32]. There are many methods of knowledge representation, such as rule-based representation, production rules, logical representation, frame representation, semantic networks, ontology representation, and so on [33-38]. Different knowledge structures can be expressed in different ways, and knowledge in the same field can also be expressed in different ways. Therefore, there is no uniform standard for knowledge representation. This paper adopts ontology representation.

Ontology is a clear formal specification of a shared conceptual model. It has four characteristics: sharing, clarity, conceptualization, and formalization [39]. Ontology is a collection of concepts, which has a certain relation and hierarchical structure. It is used to describe the inherent characteristics of things. Ontology can realize the standardized representation of knowledge, the utilization, and retrieval of knowledge, and the knowledge reasoning using defining rules. Moreover, ontology provides a special data storage mode, which has a strong ability for semantic expression and reasoning, and is suitable for information organization in the digital age. 


\subsection{Related Concepts of Knowledge Graph of Maritime Dangerous Goods}

\subsubsection{The Concepts and Entities of Maritime Dangerous Goods}

The concept of MDG $\left(C_{m d g}\right)$ refers to the class of objects reflected by various identifiable dangerous goods recorded in the IMDG Code List. It includes nine sub-concepts, which can be expressed as $C_{m d g}=\left\{C_{\text {class } 1}, C_{\text {class } 2}, \cdots, C_{\text {class } 9}\right\}$. Besides the concept of MDG, the concept set $(C)$ of KGMDG also includes the concepts of packing $\left(C_{p a}\right)$, container $\left(C_{c o n}\right)$, emergency $\left(C_{E m S}\right)$, stowage $\left(C_{s t}\right)$, segregation $\left(C_{s e}\right)$, essential attribute $\left(C_{e a}\right)$, special attribute $\left(C_{s a}\right)$, excepted quantities $\left(C_{e q}\right)$, and special provisions $\left(C_{s p}\right)$. Thus, the concept set of KGMDG can be expressed as:

$$
C=\left\{C_{m d g}, C_{p a}, C_{c o n}, C_{s e}, C_{s t}, C_{s a}, C_{e q}, C_{s p}, C_{E m s}, C_{e a}\right\}
$$

The entity of MDG $\left(E_{m d g}\right)$ refers to all distinguishable and concrete dangerous goods under the concept of MDG, that is, $E_{m d g}=\left\{E_{m d g 1}, E_{m d g 2}, \cdots E_{m d g n}\right\}, n>1$, where $E_{m d g n}$ is a certain kind of dangerous goods. In addition, there are corresponding entities under other concepts in the KGMDG. Therefore, the entity set $(E)$ of KGMDG can be expressed as:

$$
E=\left\{E_{m d g}, E_{p a}, E_{c o n}, E_{s e}, E_{s t}, E_{s a}, E_{e q}, E_{s p}, E_{E m S}, E_{e a}\right\}
$$

Table 1 shows the core concepts and some entities of KGMDG.

Table 1. The core concepts and their entities of KGMDG.

\begin{tabular}{ccc}
\hline Core Concepts $(\boldsymbol{C})$ & Sub-Concepts & Entities $(\boldsymbol{E})$ \\
\hline MDG & Class1 & flash power \\
& $\ldots$ & $\ldots$ \\
Packing requirement & Class9 & fishmeal \\
& Packing instruction & P01 \\
Container & Packing provision & PP01 \\
& Intermediate bulk containers & IBC01 \\
Essential attribute & Potable tanks and bulk containers & T1 \\
& Chemical property & flash point \\
Emergency schedules & Physical property & melting point \\
Special attribute & none & F-A, S-A \\
Stowage and handing & none & UN number \\
Segregation & none & Category A, SW1 \\
Excepted quantities & none & SG1 \\
Special provisions & none & E0 \\
& none & SP16 \\
\hline
\end{tabular}

\subsubsection{The Factual Relation of Maritime Dangerous Goods}

The relation $R$ of KGMDG represents the relationship in the knowledge of MDG. It can be divided into two categories, one is ontology common semantic relation $\left(R_{\text {ontology }}\right)$, the other is self-defined factual relations $\left(R_{\text {factual }}\right)$. Therefore, $R$ can be expressed as $R=\left\{R_{\text {ontology }}, R_{\text {factual }}\right\}$.

With the help of ontology experts and MDG experts, this paper lists the main types of relations including:

- $\quad$ kind_of $\left(R_{\text {kind_of }} \in R_{\text {ontology }}\right)$ : It is the inheritance relation between concepts, similar to the relation between superclass and subclass in object-oriented programming. For example, the concept of explosives is a sub-concept of the concept of MDG.

- instance_of $\left(R_{\text {instance_of }} \in R_{\text {ontology }}\right)$ : It is the relation between an instance and a concept. For example, "fireworks" is an instance of "explosives."

- attribute_of $\left(R_{\text {attribute_of }} \in R_{\text {ontology }}\right)$ : It denotes that a concept is an attribute of another concept. For example, the concept of essential attribute is the attribute of the concept of MDG. 
- has_a $\left(R_{\text {has_a }} \in R_{\text {factual }}\right)$ : the relation between concepts or entities.

- segregation_x $\left(R_{\text {segregation_} \_} \in R_{\text {factual }}\right)$ : It is the relation of the segregation requirement of dangerous goods. There are five segregation requirements: "exception from segregation," "away from," "separated from," "separated by a complete compartment or hold from," "separated longitudinally by an intervening complete compartment or hold from." These five requirements are expressed by "segregation_0," "segregation_1," "segregation_2," "segregation_3," and "segregation_4," respectively.

- $\quad$ stowage_position $\left(R_{\text {stowage_position }} \in R_{\text {factual }}\right)$ : It indicates the stowage location of dangerous goods.

\subsubsection{The Formalized Representation of the Knowledge Graph of Maritime Dangerous Goods}

The structure of KGMDG is shown in Figure 3. Obviously, the KGMDG can be seen as a huge semantic network graph, which consists of the conceptual layer $(C l)$, the entity layer $(E l)$, and the relation $(R)$ between the conceptual layer and entity layer, that is, $K G M D G=<C l, R, E l>$. The conceptual layer can be represented as $C l=\left\langle N_{c}, P_{c}, R_{c}\right\rangle$, where $N_{c}$ represents the concept node, $P_{c}$ represents the attribute edge, and $R_{c}$ represents the relationship between two concepts, namely, $R_{c} \subseteq N_{c} \times P_{c} \times N_{c}$. The entity layer can be represented as $E l=\left\langle N_{e}, P_{e}, R_{e}\right\rangle$, where $N_{e}$ represents the entity node or attribute value node, $P_{e}$ represents the attribute edge, and $R_{e}$ represents the relationship between two nodes. Each edge and the nodes on both sides can form a triple (subject, predicate, object). The closed dashed line in Figure 3, for example, shows a triple (A, segmentation_2, B), where subject A and object B denotes two different dangerous goods, and the predicate "segregation_2" denotes the relationship between goods A and B. This triple indicates that the segregation requirement between goods A and B is "separated from." The relation $R$ between the conceptual layer and the entity layer can be expressed using the attribute of $r d f: t y p e$, that is, $R=\left\{\left(E_{1}, r d f:\right.\right.$ type, $\left.\left.C_{1}\right) \mid E_{1} \in E, C_{1} \in C\right\}$.

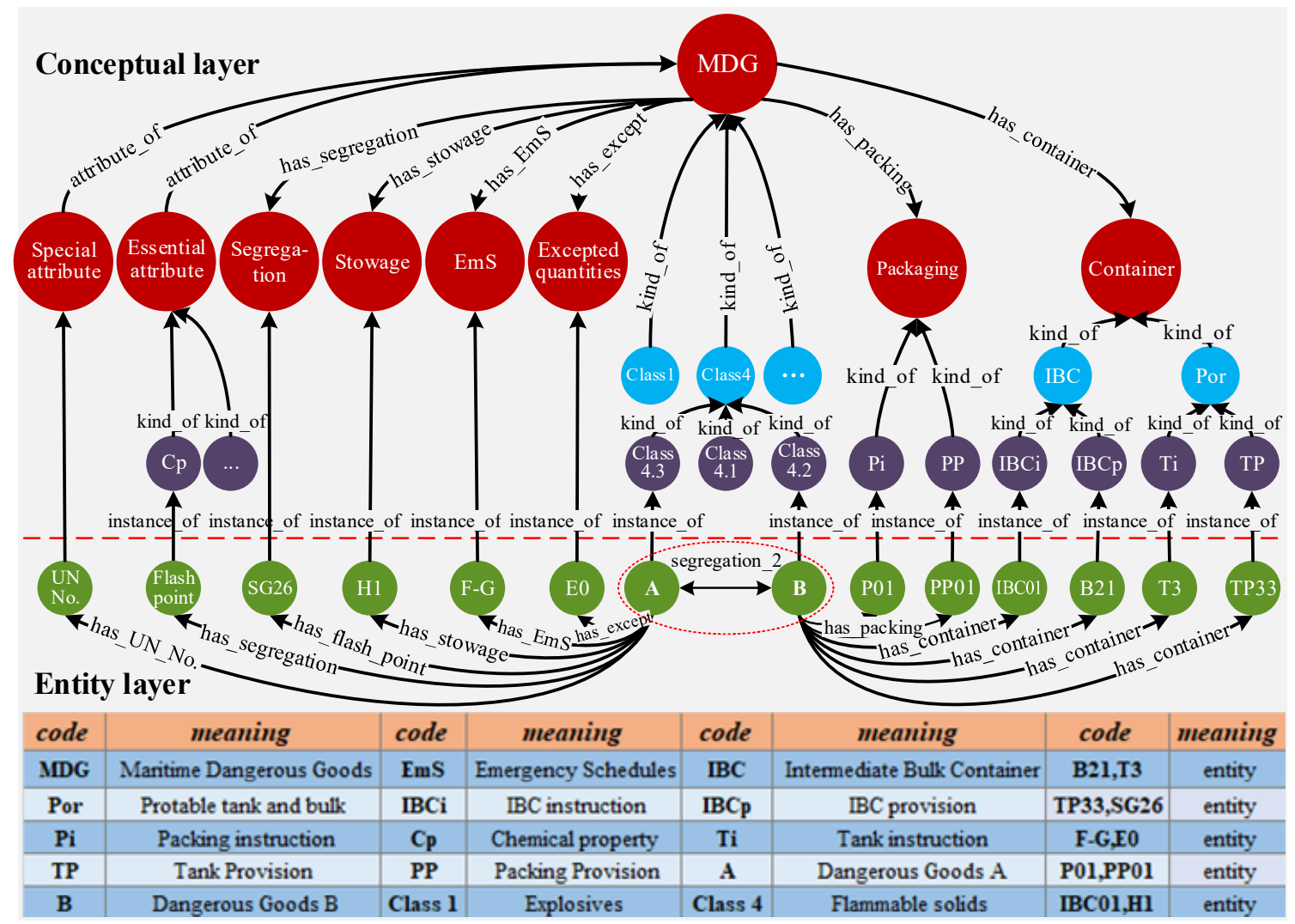

Figure 3. Structure of the KGMDG (part). 


\subsection{Construction of Knowledge Graph of Maritime Dangerous Goods}

Based on the above analysis, we propose a method for constructing the KGMDG, which consists of a conceptual layer design and an entity layer design. The specific construction processes are as follows.

\subsubsection{Conceptual Layer Design}

The conceptual layer is the frame of the knowledge graph, and it is also the core of the knowledge graph. Therefore, the task of this chapter is to construct the framework of KGMDG according to the MDG knowledge under the guidance of experts.

\section{The Determination of Core Concepts}

The core concept is the abstract analysis of the industry knowledge, which is the skeleton of the knowledge graph. The determination of core concepts is mainly centered on MDG. Operation requirements of the transportation and storage phases are core concepts, such as packaging, stowage, segregation, emergency procedures, etc. The determination of these kinds of concepts is mainly based on the IMDG Code. In addition, the MSDS is a document used by chemical manufacturers and importers to clarify the physical and chemical properties of chemicals (such as $\mathrm{PH}$ value, flash point, flammability, reactivity, etc.) and the possible hazards (such as carcinogenicity, teratogenicity, etc.) to users' health. It is an important knowledge source of MDG. Therefore, some core concepts are derived from the MSDS, including protective measures, physical and chemical properties, etc. The definition of these two kinds of concepts should be strictly examined by experts to ensure uniqueness and accuracy.

2. The Determination of Hierarchy and Relations between Concepts

The determination of hierarchy is also centered on MDG. Attribute concepts and operational concepts are placed at the same level as the concept of MDG. Then we established the relation of "attribute_of" and "has_a," respectively. The sub-concepts of MDG, packaging, and container were placed at a lower level. The relation of "kind_of" was established. Specifically, the relation between the concepts of MDG and of packaging is "has_packing," the relation between the concepts of essential attribute and MDG is "attribute_of," and the relation between the concepts of Class1 and MDG is "kind_of." The hierarchical structure was designed to meet the construction principles of ontology and try to keep it in line with the structural system of the IMDG Code.

\subsubsection{Entity Layer Design}

The task of this section is to identify entities, relations, attribute values, and other information from MDG knowledge, and to match the concepts of the conceptual layer. Specifically, the purpose is to extract knowledge and express it as a triple form of (entity, relation, entity) or (entity, attribute, attribute value).

1. Entity Extraction

Entity extraction is an important step toward constructing a knowledge graph. Its purpose is to find a set of terms or markers to represent entities or attributes from industry knowledge. After pre-processing, data in the IMDG Code List and MSDS are stored in a structured and semi-structured schema structure. Therefore, it is easy to customize the rules of entity extraction. A description of the specific operation steps of entity extraction combining with domain knowledge is as follows.

Extraction of MDG entities: The entities of MDG are the dangerous goods listed in the IMDG Code List and the MSDS. In the IMDG Code, some dangerous goods with the same proper shipping name and UN number have different operational requirements due to different packaging groups. Therefore, we need to mark the goods as different entities according to their packaging groups. As such, in the IMDG Code List, we identified the unique MDG entity according to UN numbers, proper shipping name, and packing group. Meanwhile, we took the UN number and packing group as the attribute of this entities. In the MSDS, the Chemical Abstracts Service number (CAS No.) is unique, so we extracted the unique entity based on the CAS No. 
Extraction of other entities: Different dangerous goods have different operation requirements because of their different risks and physical and chemical properties. These operational requirements have been identified as core concepts in Section 4.3.1, so we only needed to extract their entities from these core concepts. In the IMDG Code List, these entities are shown as codes, so we extracted these codes as entities.

Extraction of attribute values: According to the suggestions of experts, we extracted the important properties of transportation and storage process, including classification, subsidiary risk, limited quantities, packaging group, observations, flash point, melting point, boiling point, toxicity, etc. From the IMDG Code List, we extracted classification, subsidiary risk, limited quantities, packaging groups, and observations as attributes, and extracted their number or strings as attribute values. In the MSDS, we extracted flash points, melting points, boiling points, toxicity, and other attributes, and extract their values as attribute values.

\section{Entity Alignment}

Entity alignment was used to solve the problem that multiple entities extracted from different data sources pointed to the same object. One kind of dangerous good has a proper shipping name in the IMDG Code, while the chemical name is labeled in the MSDS, and the two names may be inconsistent. In KGMDG, we mainly focused on MDG in the IMDG Code, so we aligned dangerous goods entities according to UN numbers and packaging groups. Taking dangerous goods with UN number 1307 as an example, it has only one name in the IMDG Code as "xylene," but there are three different chemical names in MSDS: "1,2-xylene," "1,3-xylene," and "1,4-xylene." According to our entity alignment rules, these three entities extracted from MSDS are treated as one kind of dangerous goods.

\section{Entity Relations}

According to the relations between concepts, the relation between MDG entities and other entities is "has_a," the relation between MDG entity and attribute value is "has_attribute," the relation between MDG entities is "segregation_ $x$," and the relation between entities and their concepts is "instance_of."

So far, we have identified entities, attributes, attribute values, and relations. Then, we transformed the MDG knowledge into triple forms of (entity, relation, entity) or (entity, attribute, attribute value). Some triples in the KGMDG are shown in Table 2.

Table 2. Some subject-predicate-object triples in KGMDG.

\begin{tabular}{ccc}
\hline Subject & Predicate & Object \\
\hline MDG & has_EmS & EmS \\
flash point & attribute_of & MDG \\
Class1 & kind_of & MDG \\
SG26 & instance_of & Segregation \\
Dangerous Goods A & instance_of & Class4.3 \\
Dangerous Goods A & segregation_2 & Dangerous Goods B \\
Dangerous Goods B & has_packing & P01 \\
Dangerous Goods B & has_container & B21 \\
\hline
\end{tabular}

\section{Relational Reasoning}

Relational reasoning is mainly based on the existing relations in the knowledge base to obtain other relations by reasonable rules. The reasoning rules used by the KGMDG are mainly the description logic (DL) [40] and the semantic web rule language rule (SWRL) [41].

The DL system consists of a knowledge base and reasoning services. The knowledge base consists of two parts: Tbox and Abox. Tbox usually describes concepts and relationships in the form of terminological axioms. There are two forms of terminological axioms:

- inclusion: $C \sqsubseteq D$ or $R \sqsubseteq S$, for example, Explosives $\sqsubseteq M D G$

- equality: $C \equiv D$ or $R \equiv S$, for example, Flammable gases $\equiv$ Gases $\sqcap \exists$ hasExplosion.MDG

where $C, D$ are the concepts and $R, S$ are the relations. Abox is an instantiated assertion of individuals and relations. There are two forms of instantiated assertion: 
- Conceptual assertion $C(a)$ : The individual $a$ is an instance of concept $C$. "Flammable gases(propylene)," for example, declares that propylene is an instance of the concept of flammable gases.

- Relational assertion $R(a, b)$ : The relationship between individual $a$ and $b$ is $R$. For example, "has_packing(propylene, P200)" declares that propylene needs to be packed according to the requirements of P200.

DL can provide reasoning services such as consistency, satisfiability, subsumption, instance checking, etc. By describing concepts or entities in ontology, the system can automatically determine whether a concept is a subclass of another concept or whether a concept is compatible with existing concepts after running DL rules. For example, by defining the concept of MDG and the concept of flammable gases, we can get that flammable gases are subclasses of MDG after running DL rules.

The semantic web rule language (SWRL) is a proposed language for the semantic web that can be used to express rules as well as logic, combining Web Ontology Language (OWL) DL or OWL Lite with a subset of the rule markup language [41]. In KGMDG, the SWRL is mainly used to determine the stowage and segregation requirements. Transforming the stowage and segregation rules in the IMDG Code into the SWRL can automatically determine the segregation requirements between different dangerous goods. For example, we constructed triples of (Ammonium picrate, instance_of, MDG), (Ammonium picrate, has_segregation, SG7), and (Methanol, instance_of, Class3). We convert the segregation code of SG7 into:

$$
\operatorname{MDG}(? a)^{\wedge} \text { has_segregation(?a,SG7)`Class3(?b)- > segregation_1(?a,?b) }
$$

where SG7 means "stow 'away from' Class 3". In this rule, "“" means "and," and "->" means reasoning; "MDG(?a)" means "a" is an entity of the concept of MDG; similarly, "Class3(?b)" means " $b$ " is an entity of the concepts of category III of MDG; "has_segregation(?a, SG7)" means that entity "a" has a segregation code of SG7; and "segregation_1 (?a, ?b)" means that the segregation requirement between entity $a$ and $b$ is "segregation_1." Finally, by running the reasoning engine, we get the triple of (Ammonium picrate, segregation_1, Methanol), that is, the segregation requirement of Ammonium picrate and Methanol is "away from." We define SWRL for the segregation rules and stowage rules respectively in the ontology. Table 3 shows some SWRL in the ontology.

Table 3. Some SWRL in the ontology.

\begin{tabular}{|c|c|c|}
\hline Rule Name & Rule & Explanation \\
\hline StA & MDG(?a)^ has_stowage(?a, StA) -> stowage_position(?a, “on deck”) & Stow on deck \\
\hline GS01 & Class1_1(?a)^Class2_1(?b) -> segregation_4(?a,?b) & Class1.1 "segregation_4" Class2.2 \\
\hline GS13 & Class7(?a)^Class8(?b) -> segregation_2(?a, ?b) & Class7 "segregation_2" Class8 \\
\hline SG7 & $\operatorname{MDG}(? a) \wedge$ has_segregation(?a, SG7)^Class3(?b) -> segregation_1(?a, ?b) & Stow "segregation_1" Class3 \\
\hline SG15 & $\operatorname{MDG}(? a)^{\wedge}$ has_segregation(?a, SG15) ^Class3(?b) -> segregation_2(?a, ?b) & Stow "segregation_2" Class3 \\
\hline SG35 & $\operatorname{MDG}(? a) \wedge$ has_segregation(?a, SG29) ^Acid(?b) -> segregation_2(?a, ?b) & Stow "segregation_2" acids \\
\hline SG46 & $\operatorname{MDG}(? \mathrm{a})^{\wedge}$ has_segregation(?a, SG46)^Chlorine(?b) -> segregation_2(?a, ?b) & Stow "segregation_2" chlorine \\
\hline SG63 & MDG(?a)^ has_segregation(?a, SG63)^Class1(?b) -> segregate_4(?a, ?b) & Stow "segregation_4" Class1 \\
\hline SG65 & $\begin{array}{c}\mathrm{MDG}(? \mathrm{a}) \wedge \text { has_segregation }(? \mathrm{a}, \mathrm{SG} 65)^{\wedge} \text { Class1 }(? \mathrm{~b}) \wedge \text { different_from }(? \mathrm{~b}, ? \mathrm{c}) \wedge \\
\text { Class1_4(?c) -> segregation_3(?a, ?b) }\end{array}$ & $\begin{array}{c}\text { Stow "segregation_3" Class1 except } \\
\text { for division1.4 }\end{array}$ \\
\hline
\end{tabular}

\section{Results and Discussion}

Based on the above analysis, we have completed the construction of a KGMDG. Below we show the visualization, professional knowledge retrieval, and segregation requirements judgement based on a knowledge graph.

\subsection{Knowledge Visualization}

The KGMDG provides a graphical display function. As can be seen from Figure 4, the KGMDG does not only visualize the concepts, entities, the relations between concepts and the relations between entities, but also realizes the visualization of complex knowledge systems. It can establish a relation between "knowledge islands," and thus it can improve the relation between these islands. In this way, it is helpful to discover the potential relations between knowledge and to excavate implicit knowledge. 


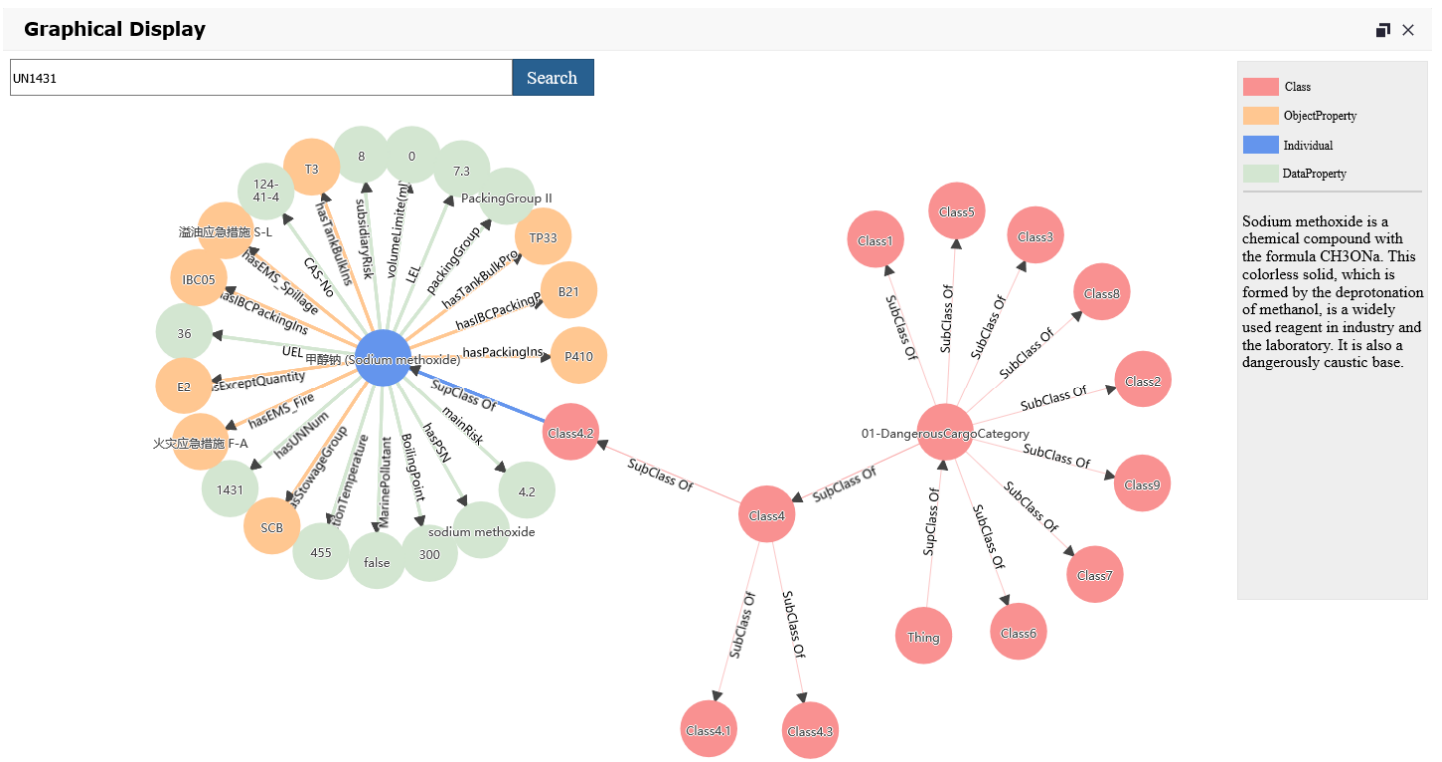

Figure 4. Visual schematic diagram of MDG knowledge (taking "sodium methoxide" as an example). Here the users can click on the target node to display the specific content of this node and double-click on the target node to display the nodes directly associated with the target node.

\subsection{Knowledge Retrieval}

The knowledge graph makes the form of knowledge retrieval change from being traditional text-based to concept-based, which makes the knowledge graph more substantial, semantic, and intelligent, and also improves the efficiency and quality of the knowledge retrieval. Based on the knowledge graph, not only can the relevant knowledge of user queries be displayed accurately and in detail, but also the classification navigation of knowledge can be realized to help users locate the needed knowledge quickly. Figure 5 shows the search page of methanol, it shows some important physical and chemical properties and operation requirements. This query system can provide the professional knowledge that the user desires, and it also extended the information of the user query.

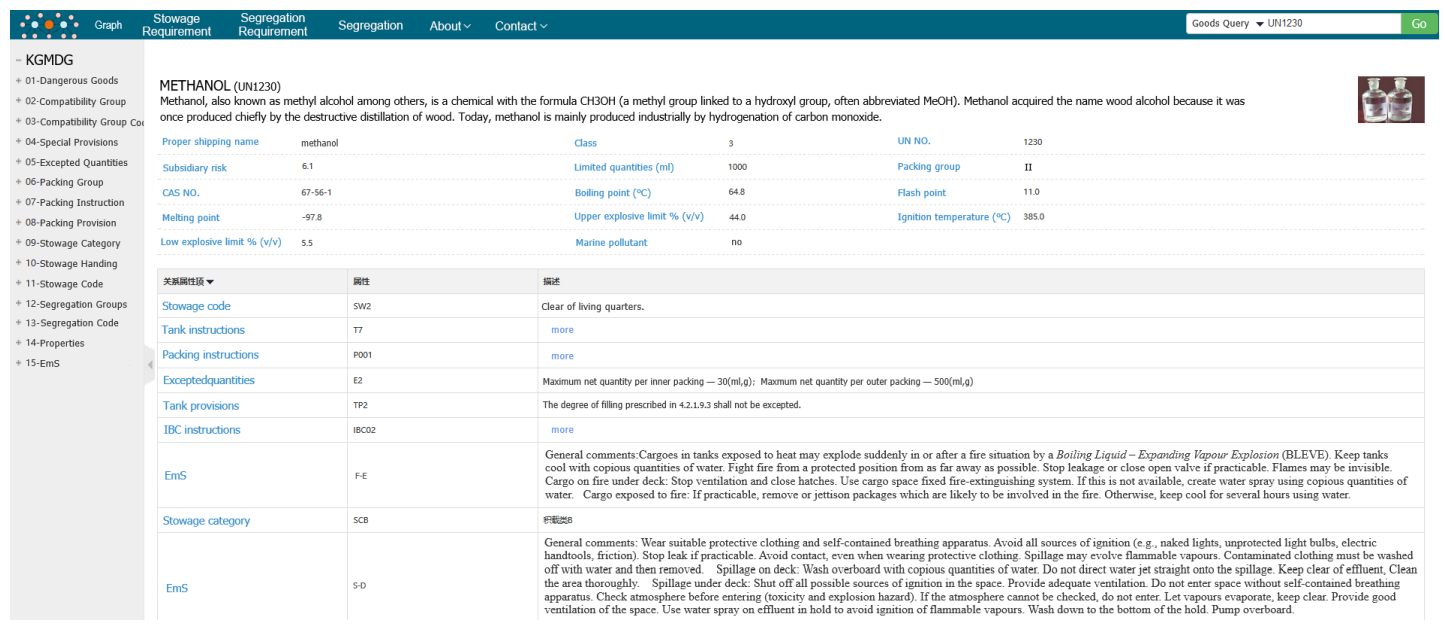

Figure 5. Knowledge retrieval diagram of KGMDG (taking "methanol" as an example). The left side is the navigation bar of this system, which can quickly and easily locate the relevant concepts and entities. The right side is the display interface of professional knowledge, in which the proper shipping name, UN number, brief introduction, picture, and some important attributes are shown above, and the operation requirements are shown below, including stowage, segregation, packaging, etc. 


\subsection{Automatic Judgement of the Segregation Requirement}

Based on the reasoning function of the knowledge graph, the segregation requirement of dangerous goods can be automatically realized. In the loading work, the judgement process of the segregation requirement of MDG is complex, as shown in Figure 6. First, we needed to judge whether there was an exemption clause for these two goods. Then, we judged whether there is a segregation code for the two goods. If there is a segregation code, then it needs to be judged according to the requirements of the segregation code. If there is no segregation code for both dangerous goods, it needs to be judged by the general segregation table in the IMDG Code. Finally, the segregation requirement is obtained. In the KGMDG, we transformed the above process into SWRL, which can realize the automatically judgement of segregation requirements. Tacking the segregation requirement of magnesium powder and acrylic acid as an example, there are no exemption clauses for both goods. Then, we found that magnesium powder has the segregation code "SG35". "SG35" means "stow 'separated from' acids". Acrylic acid is an entity of acids, so the result is "separated from." The SWRL of SG35 is "MDG(?X) ^ has_segregation (?X, SG35) ^Acids(?Y) -> segregation_2(?X, ?Y)”. Figure 7 shows the KGMDG's judgment on the segregation requirements of these two goods.

In addition, we can also judge the segregation requirements of one kind of dangerous goods. We turned all the segregation rules in IMDG Code into SWRL. By entering one kind of goods, we could get all the goods that needed to be separated from it. Figure 8 shows some dangerous goods that need to be separated from acrylic acid.

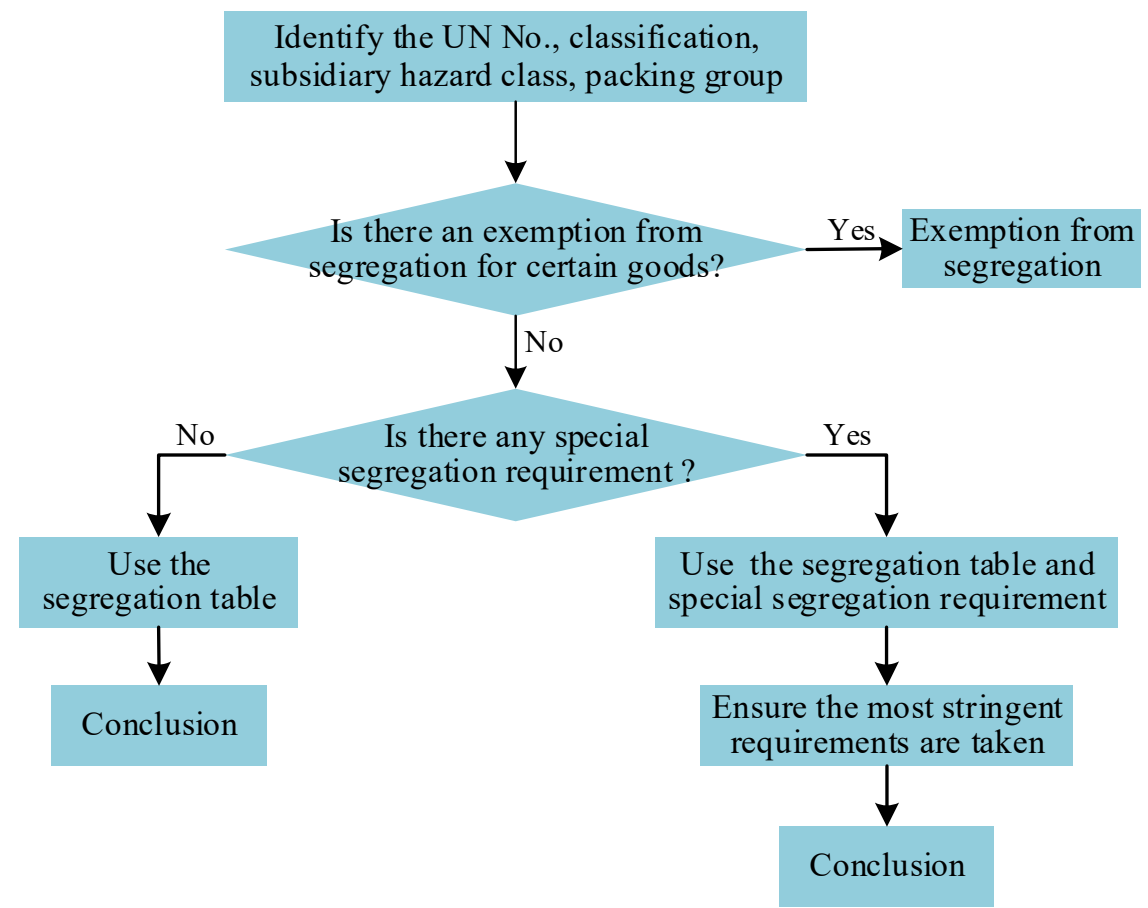

Figure 6. Flow chart of segregation requirement. 
Segregation Requirement

UN1418

Separated from: In different compartments or hold when stowed under deck. Provided the intervening deck is resistant to fire and liquid, a vertical separation, i.e., in different compartments, may be accepted as equivalent to this segregation. For on deck stowage, this segregation means a separation by a distance of at least $6 \mathrm{~m}$ horizontally.

\section{Separated from}

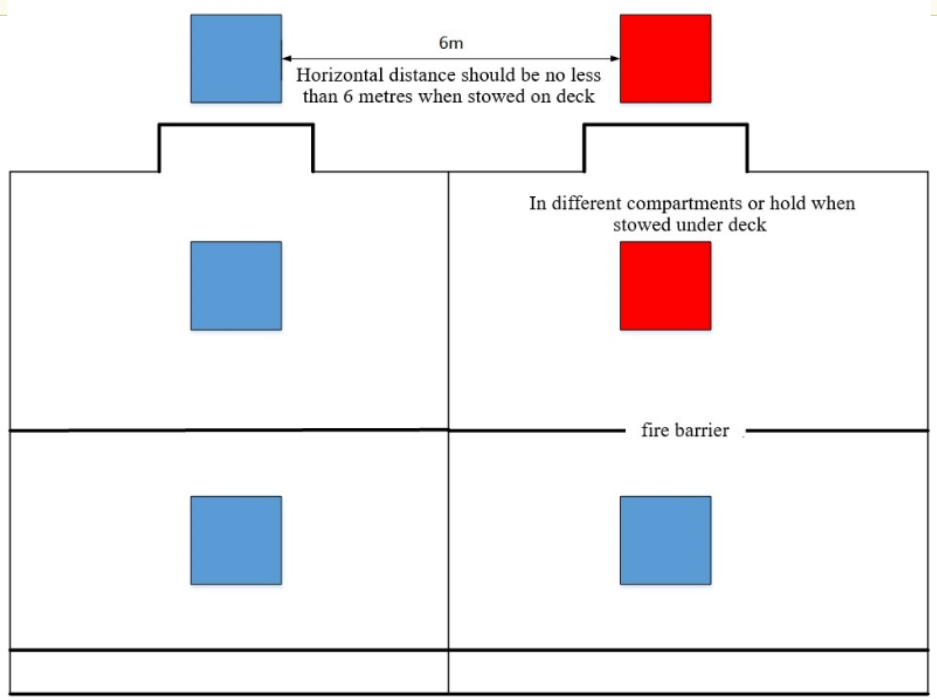

Figure 7. Diagram of segregation requirement judgment (taking magnesium powder and acrylic acid as an example).

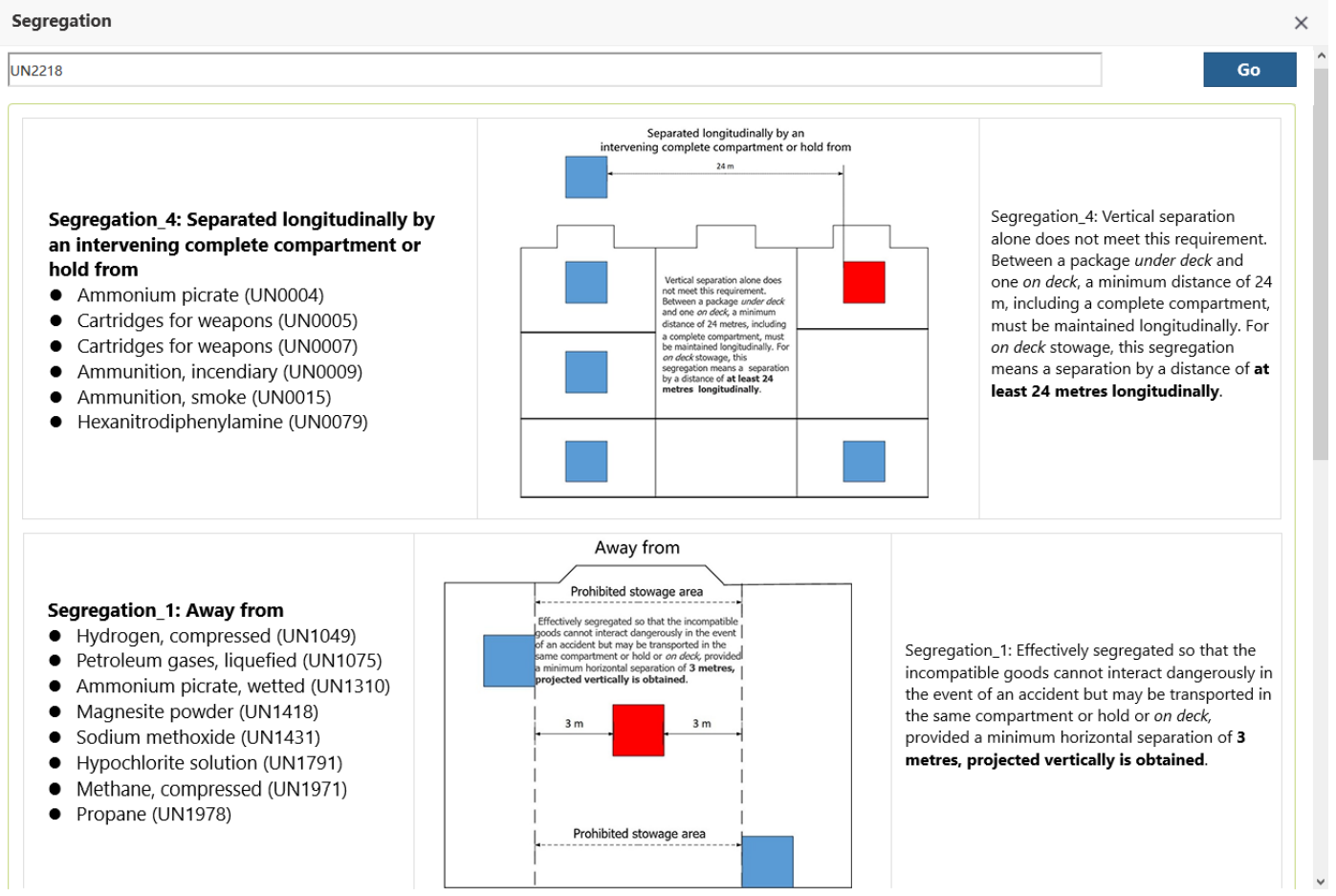

Figure 8. Diagram of segregation requirement of one kind of goods (taking acrylic acid as an example). 


\section{Conclusions}

This paper introduced the technology of a knowledge graph into the field of MDG and established the KGMDG, a new technology including knowledge organization, management, and service. The KGMDG realizes the interrelation of MDG knowledge and displays the knowledge hierarchy of industry knowledge quickly. Compared with traditional knowledge management technology, a knowledge graph displays knowledge more visually and achieves more efficiency and higher quality knowledge retrieval. The KGMDG provides a new method for MDG knowledge management and mining. It is of great significance to promote the sharing, dissemination, and utilization of knowledge. However, there is still a lot of knowledge that is unclear in the transportation of dangerous goods, such as how to better express this knowledge and facilitate computer understanding.

Author Contributions: The author Q.Z. drafted the manuscript. Y.W. and C.Z. contributed to the research methodology. H.L. and D.H. contributed to the visualization. F.Z. and C.X. reviewed and polished the language.

Funding: This research was funded by the National Science Foundation of China (NSFC) (Project Number: 51679180), the National Key R\&D Program of China (Project Number: 2018YFC1407405), the National Key R\&D Program of China (Project Number: 2018YFC0213900), the National Science Foundation of China (NSFC) (Project Number: 51709218).

Conflicts of Interest: The authors declare no conflict of interest.

\section{References}

1. Mullai, A. A Risk Analysis Framework for Maritime Transport of Packaged Dangerous Goods—A Validating Demonstration Volume II; Engineering Logistics, Lund Institute of Technology, Lund University: Lund, Sweden, 2007.

2. Ellis, J. Undeclared dangerous goods—Risk implications for maritime transport. WMU J. Marit. Aff. 2010, 9, 5-27. [CrossRef]

3. Ellis, J. Analysis of accidents and incidents occurring during transport of packaged dangerous goods by sea. Saf. Sci. 2011, 49, 1231-1237. [CrossRef]

4. IMO. IMDG Code. International Maritime Dangerous Goods Code; International Maritime Organization: London, UK, 2014.

5. Gruber, T. Ontology. In Encyclopedia of Database Systems; Liu, L., ÖZsu, M.T., Eds.; Springer: New York, NY, USA, 2009; ISBN 978-0-387-35544-3.

6. Berners-Lee, T.; Hendler, J. The Semantic Web. Sci. Am. 2002, 284, 28-37. [CrossRef]

7. Chau, K.W. An ontology-based knowledge management system for flow and water quality modeling. Adv. Eng. Softw. 2007, 38, 172-181. [CrossRef]

8. Ding, L.Y.; Zhong, B.T.; Wu, S.; Luo, H.B. Construction risk knowledge management in BIM using ontology and semantic web technology. Saf. Sci. 2016, 87, 202-213. [CrossRef]

9. Zhang, C.; Romagnoli, A.; Zhou, L.; Kraft, M. Knowledge management of eco-industrial park for efficient energy utilization through ontology-based approach. Appl. Energy 2017, 204, 1412-1421. [CrossRef]

10. Antezana, E.; Kuiper, M.; Mironov, V. Biological knowledge management: The emerging role of the Semantic Web technologies. Brief. Bioinform. 2009, 10, 392-407. [CrossRef] [PubMed]

11. Singhal, A. Introducing the Knowledge Graph: Things, Not Strings. Available online: https://www.blog. google/products/search/introducing-knowledge-graph-things-not/ (accessed on 16 May 2012).

12. Yu, T.; Li, J.; Yu, Q.; Tian, Y.; Shun, X.; Xu, L.; Zhu, L.; Gao, H. Knowledge graph for TCM health preservation: Design, construction, and applications. Artif. Intell. Med. 2017, 77, 48-52. [CrossRef] [PubMed]

13. Qi, G.; Gao, H.; Wu, T. The research advances of knowledge graph. Technol. Intell. Eng. 2017, 3, 4-25.

14. Yan, J.; Lv, T.; Yu, Y. Construction and recommendation of a water affair knowledge graph. Sustainability 2018, 10, 3429. [CrossRef]

15. Lam, J. Rough Set Approach to Marine Cargo Risk Analysis. In Proceedings of the International Forum on Shipping, Ports and Airports (IFSPA), Hong Kong, China, 27-30 May 2012.

16. Liu, X.; Li, J.; Li, X. Study of dynamic risk management system for flammable and explosive dangerous chemicals storage area. J. Loss Prev. Process Ind. 2017, 49, 983-988. [CrossRef]

17. Yang, Q.; Chin, K.S.; Li, Y.L. A quality function deployment-based framework for the risk management of hazardous material transportation process. J. Loss Prev. Process Ind. 2018, 52, 81-92. [CrossRef] 
18. Lieggio Junior, M.; Granemann, S.R.; de Souza, O.A.; Rocha, C.H. Transportation of dangerous goods by road: The Brazilian case for selection of carriers based on a risk management methodology. Transp. Plan. Technol. 2012, 35, 677-696. [CrossRef]

19. Van der Vlies, V.A. Qualitative approach to risk management of hazardous materials in the Netherlands: Lessons learned from 7 sluice cases. J. Risk Res. 2015, 18, 947-964. [CrossRef]

20. Lehmann, J.; Isele, R.; Jakob, M.; Jentzsch, A.; Kontokostas, D.; Mendes, P.N.; Hellmann, S.; Morsey, M.; Van Kleef, P.; Auer, S.; et al. DBpedia-A large-scale, multilingual knowledge base extracted from Wikipedia. Semant. Web 2015, 6, 167-195.

21. Rebele, T.; Suchanek, F.; Hoffart, J.; Biega, J.; Kuzey, E.; Weikum, G. YAGO: A multilingual knowledge base from wikipedia, wordnet, and geonames. In Proceedings of the 15th International Semantic Web Conference, Kobe, Japan, 17-21 October 2016.

22. Bollacker, K.; Evans, C.; Paritosh, P.; Sturge, T.; Taylor, J. Freebase: A collaboratively created graph database for structuring human knowledge. In Proceedings of the 2008 ACM SIGMOD International Conference on Management of Data, Vancouver, BC, Canada, 9-12 June 2008.

23. Niu, X.; Sun, X.; Wang, H.; Rong, S.; Qi, G.; Yu, Y. Zhishi.me-Weaving Chinese linking open data. In $e$ Lecture Notes in Computer Science (Including Subseries Lecture Notes in Artificial Intelligence and Lecture Notes in Bioinformatics); Springer: Berlin, Germany, 2011.

24. Xu, B.; Xu, Y.; Liang, J.; Xie, C.; Liang, B.; Cui, W.; Xiao, Y. CN-DBpedia: A never-ending chinese knowledge extraction system. In Lecture Notes in Computer Science (Including Subseries Lecture Notes in Artificial Intelligence and Lecture Notes in Bioinformatics); Springer: Berlin, Germany, 2017.

25. Ernst, P.; Siu, A.; Weikum, G. KnowLife: A versatile approach for constructing a large knowledge graph for biomedical sciences. BMC Bioinform. 2015, 16, 167. [CrossRef]

26. Rotmensch, M.; Halpern, Y.; Tlimat, A.; Horng, S.; Sontag, D. Learning a Health Knowledge Graph from Electronic Medical Records. Sci. Rep. 2017, 7, 5994. [CrossRef] [PubMed]

27. Jia, L.; Liu, J.; Tong, Y.U.; Dong, Y.; Zhu, L.; Gao, B.; Liu, L. Construction of Traditional Chinese Medicine Knowledge Graph. J. Med. Inform. 2015, 8, 51-53.

28. Miao, F.; Liu, H.; Huang, Y.; Liu, C.; Wu, X. Construction of Semantic-Based Traditional Chinese Medicine Prescription Knowledge Graph. In Proceedings of the 2018 IEEE 3rd Advanced Information Technology, Electronic and Automation Control Conference (IAEAC), Chongqing, China, 12-14 October 2018.

29. Liu, P.; Wang, X.; Sun, X.; Shen, X. HKDP: A Hybrid Knowledge Graph Based Pediatric Disease Prediction System. In Proceedings of the International Conference on Smart Health, Wuhan, China, 1-3 July 2018; pp. 78-90.

30. Wang, R.; Yan, Y.; Wang, J.; Jia, Y.; Zhang, Y.; Zhang, W.; Wang, X. AceKG: A Large-scale Knowledge Graph for Academic Data Mining. In Proceedings of the CIKM'18 27th ACM International Conference on Information and Knowledge Management, Torino, Italy, 22-26 October 2018; pp. 1487-1490.

31. Chen, P.; Lu, Y.; Zheng, V.W.; Chen, X.; Yang, B. KnowEdu: A System to Construct Knowledge Graph for Education. IEEE Access 2018, 6, 31553-31563. [CrossRef]

32. Davis, E. Knowledge Representation. In International Encyclopedia of the Social E Behavioral Sciences, 2nd ed.; Elsevier: Amsterdam, The Netherlands, 2015; ISBN 9780080970875.

33. Zhang, Y.; Luo, X.; Zhao, Y.; Zhang, H.C. An ontology-based knowledge framework for engineering material selection. Adv. Eng. Inform. 2015, 29, 985-1000. [CrossRef]

34. Nazaruks, V.; Osis, J. A Survey on Domain Knowledge Representation with Frames. In Proceedings of the 12th International Conference on Evaluation of Novel Approaches to Software Engineering (ENASE 2017), Porto, Portugal, 28-30 April 2017; pp. 346-354.

35. Xu, Z.; Liu, Y.; Mei, L.; Hu, C.; Chen, L. Semantic based representing and organizing surveillance big data using video structural description technology. J. Syst. Softw. 2015, 102, 217-225. [CrossRef]

36. Braffort, A.; Filhol, M.; Delorme, M.; Bolot, L.; Choisier, A.; Verrecchia, C. KAZOO: A sign language generation platform based on production rules. Univers. Access Inf. Soc. 2016, 15, 541-550. [CrossRef]

37. Liu, H.; Gegov, A.; Cocea, M. Rule Based Networks: An Efficient and Interpretable Representation of Computational Models. J. Artif. Intell. Soft Comput. Res. 2017, 7, 111-123. [CrossRef]

38. Cresswell, M.J. Logics and Languages; Routledge: Abington, Thames, UK, 2017.

39. Eriksson, O.; Johannesson, P.; Bergholtz, M. Institutional ontology for conceptual modeling. J. Inf. Technol. 2018, 33, 105-123. [CrossRef] 
40. Nardi, D.; Brachman, R.J. An Introduction to Description Logics. In The Description Logic Handbook; Cambridge University Press: Cambridge, UK, 2010; ISBN 9781139025355.

41. Horrocks, I.; Patel-schneider, P.F.; Boley, H.; Tabet, S.; Grosof, B.; Dean, M. SWRL: A Semantic Web Rule Language Combining OWL and RuleML. W3C Memb. Submiss. 2004, 21, 1-31.

(C) 2019 by the authors. Licensee MDPI, Basel, Switzerland. This article is an open access article distributed under the terms and conditions of the Creative Commons Attribution (CC BY) license (http://creativecommons.org/licenses/by/4.0/). 